\title{
New Modelling Capabilities in Commercial Software for High-Gain Antennas
}

Jørgensen, Erik; Lumholt, Michael ; Meincke, Peter; Zhou, Min; Sørensen, Stig B. ; Borries, Oscar ; Cappellin, Cecilia; Frandsen, Poul Erik

Published in:

2012 6th European Conference on Antennas and Propagation

Link to article, DOI:

10.1109/EuCAP.2012.6206621

Publication date:

2012

Document Version

Publisher's PDF, also known as Version of record

Link back to DTU Orbit

Citation (APA):

Jørgensen, E., Lumholt, M., Meincke, P., Zhou, M., Sørensen, S. B., Borries, O., Cappellin, C., \& Frandsen, P. E. (2012). New Modelling Capabilities in Commercial Software for High-Gain Antennas. In 20126 th European Conference on Antennas and Propagation (pp. 855-859). IEEE. https://doi.org/10.1109/EuCAP.2012.6206621

\section{General rights}

Copyright and moral rights for the publications made accessible in the public portal are retained by the authors and/or other copyright owners and it is a condition of accessing publications that users recognise and abide by the legal requirements associated with these rights.

- Users may download and print one copy of any publication from the public portal for the purpose of private study or research.

- You may not further distribute the material or use it for any profit-making activity or commercial gain

- You may freely distribute the URL identifying the publication in the public portal 


\title{
New Modelling Capabilities in Commercial Software for High-Gain Antennas
}

\author{
Erik Jørgensen, Michael Lumholt, Peter Meincke, Min Zhou, Stig B. Sørensen, \\ Oscar Borries, Cecilia Cappellin, and Poul Erik Frandsen \\ TICRA \\ Læderstræde 34, DK-1201 Copenhagen \\ Denmark \\ Email: \{ej,ml,pme,mz,sbs,ob,cc,pef $\} @$ ticra.com
}

\begin{abstract}
This paper presents an overview of selected new modelling algorithms and capabilities in commercial software tools developed by TICRA. A major new area is design and analysis of printed reflectarrays where a fully integrated design environment is under development, allowing fast and accurate characterization of the reflectarray element, an initial phaseonly synthesis, followed by a full optimization procedure taking into account the near-field from the feed and the finite extent of the array. Another interesting new modelling capability is made available through the DIATOOL software, which is a new type of EM software tool aimed at extending the ways engineers can use antenna measurements in the antenna design process. The tool allows reconstruction of currents and near fields on a 3D surface conformal to the antenna, by using the measured antenna field as input. The currents on the antenna surface can provide valuable information about the antenna performance or undesired contributions, e.g. currents on a cable, can be artificially removed. Finally, the CHAMP software will be extended to cover reflector shaping and more complex materials, which combined with a much faster execution speed will make CHAMP an ideal tool for design and optimization of compact rotationally symmetric reflector systems, e.g., maritime satellite terminals.
\end{abstract}

\section{INTRODUCTION}

TICRA offers a range of dedicated EM modelling tools that each represents the commercial state-of-the-art in their area. The tools are aimed at expert users that require high speed and ease-of-use, which can only be reached by a tool targeted for a specific antenna type and application area. This contribution discusses recent developments and extended modelling capabilities in three selected software tools and application areas: Analysis and optimization of reflectarrays, antenna diagnostics and processing of measured fields in DIATOOL, and synthesis of rotationally symmetric high-gain reflector systems using CHAMP.

\section{ANALysis AND Design of Printed ReflectarRays}

Analysis of reflectarrays is a major new modelling capability under development. Currently available commercial tools allow evaluation of the single-element phase response in a periodic environment but the remaining tasks needed in the design of reflectarrays are typically not addressed. The typical design process is then limited to a phase-only synthesis of the element sizes and a subsequent evaluation of the reflectarray radiation pattern, often using a general full-wave tool leading to a computation time measured in hours. This process often leads to sub-optimal designs or requires substantial in-house software developments by the antenna designer. TICRA's reflector antenna tools will soon be enhanced with an accurate and fast analysis technique for printed reflectarrays, which is not limited to extraction of the single element phase response. The reflectarray analysis will be integrated in the general purpose reflector software GRASP, which greatly enhances the possible modelling scenarios. Typical examples include combinations of solid reflectors and reflectarrays, high-level feed models or import of a measured feed field, as well as accurate evaluation of undesired effects such as strut scattering or scattering in the feed structure.

The reflectarray analysis is based on a higher-order spectraldomain Method of Moments suitable for periodic structures. The basis functions are higher-order Legendre basis functions [1], which have two distinct advantages in comparison with typical low-order functions: The number of unknowns is reduced and the large support in the spatial domain leads to a narrow Fourier spectrum, thus reducing the number of Floquet modes required for a given accuracy. In addition, the algorithm employs an accurate radiation pattern evaluation [2], which also allows the finite extent of the array to be included. These new developments result in a method that is fast enough for optimization, while still maintaining a high degree of accuracy, which is often comparable to the accuracy obtained by full-wave solvers. The performance of the algorithm has been tested on the reflectarray sample shown in Figure 1. The radiation patterns were measured at the DTUESA Spherical Near-Field Test Facility and a comparison between the measured and the predicted pattern is shown in Figure 2, where an excellent agreement can be observed. 
The new capabilities will allow an improved reflectarray design procedure involving the following steps:

1) Characterization of the reflectarray element phase response using the fast and accurate algorithm described above.

2) Initial phase-only synthesis.

3) Full optimization of the element sizes, taking into account both amplitude and phase, the finite extent of the array, actual incidence angle, and possibly irregular grid arrangement.

4) Evaluation of parasitic effects, such as strut scattering and feed blockage.

Steps 1-3 are performed automatically by the software, which greatly enhances the productivity of the antenna designer. The software allows reflectarrays with irregular grids and the optimization procedure determines the optimal grid arrangement. An example reflectarray optimized for a contoured beam coverage over Europe is shown in Figure 3, see [3] for details.

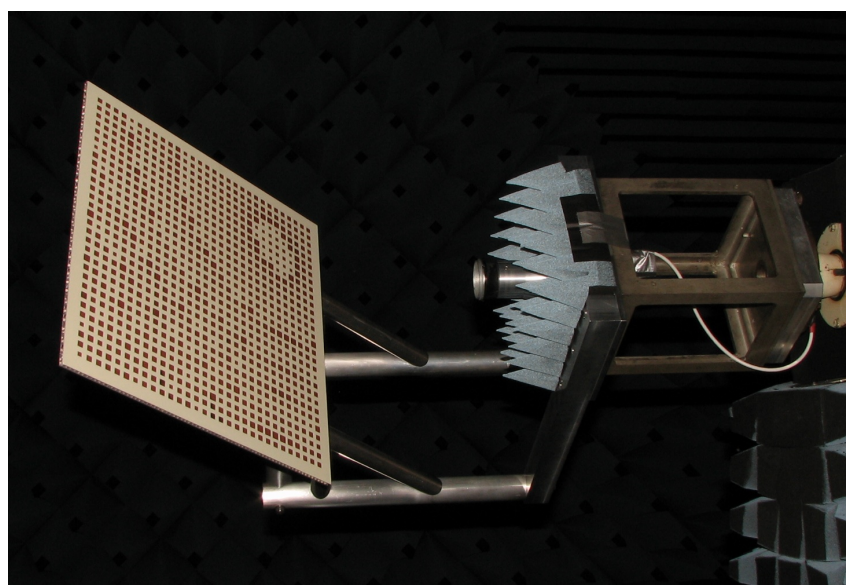

Fig. 1. Measurement setup for reflectarray sample.

\section{Processing of Measured Fields using DiATOOL}

The recent scientific developments in the area of inversesource methods have enabled the introduction of a new kind of commercial EM modelling tool. TICRA has released the software package DIATOOL, which performs a 3D reconstruction of conformal antenna currents based on measured fields. This capability opens up a range of applications, e.g., antenna diagnostics where errors can be located by inspection of the reconstructed surface currents. In addition, the software allows engineers to perform advanced pattern filtering by artificial removal of undesired features, such as cancellation of currents flowing on support structures or cables. The innovative 3D source reconstruction method is based on the higher-order inverse Method of Moments described in [4]-[5].

DIATOOL guides the user through the following phases in the reconstruction process:

1) Import of measured fields through one of several supported file formats, including formats compatible with major vendors of measurement ranges, e.g., MI Technologies and NSI.

2) Definition of the reconstruction surface. Several simple built-in models are available (boxes, spheres, cylinders, etc.) but arbitrary shapes may be imported as CAD files or as a meshed geometry.

3) Definition of special properties on some parts of the reconstruction surface, e.g., perfect conductivity, a zerofield condition, or zero radiation for filtering purposes.

4) Definition of additional derived outputs, e.g., filtered noise-free patterns radiated by the reconstructed currents.

The DIATOOL software has been validated through several practical cases reported in [6], [7]. An additional example involving a typical antenna diagnostics procedure is reported below. The antenna under test is the $6 \times 7$ slot array shown in Figure 4, suffering from two independent problems: A row-wise excitation problem of the slots and an intentionally covered slot. The radiation pattern was measured at the DTUESA Spherical Near-Field Test Facility and used as input to the DIATOOL software. The amplitude and phase of the copolarized field on the top surface are reported in Figure 4 where slot rows 2 and 3 appear with an unexpected strong excitation, thus clearly revealing the excitation problem. The covered slot can also be identified, in particular in the phase plot where the a distinct phase difference is observed between the area containing the covered slot and the surrounding area.

\section{DESIGN AND Optimization OF Rotationally SYMMETRIC REFLECTOR SYSTEMS}

The emerging satellite services in Ka-band have spawned a considerable interest in rotationally symmetric compact reflector systems. These compact systems often include a very tight integration of feed, subreflector, and main reflector, or alternatively a single-reflector system with a backward radiating feed of the top-hat type. The tight integration of the system components and the common use of dielectric support structures imply that an accurate full-wave model is needed. At the same time, the electrical size of the configuration makes it impossible to optimize the antenna performance using a general full-wave software package. CHAMP is a dedicated software package for design of corrugated feeders and is widely recognized for it's speed and accuracy. The horn analysis is based on mode-matching, which enables analysis of even complex corrugated feed geometries in a few seconds. 


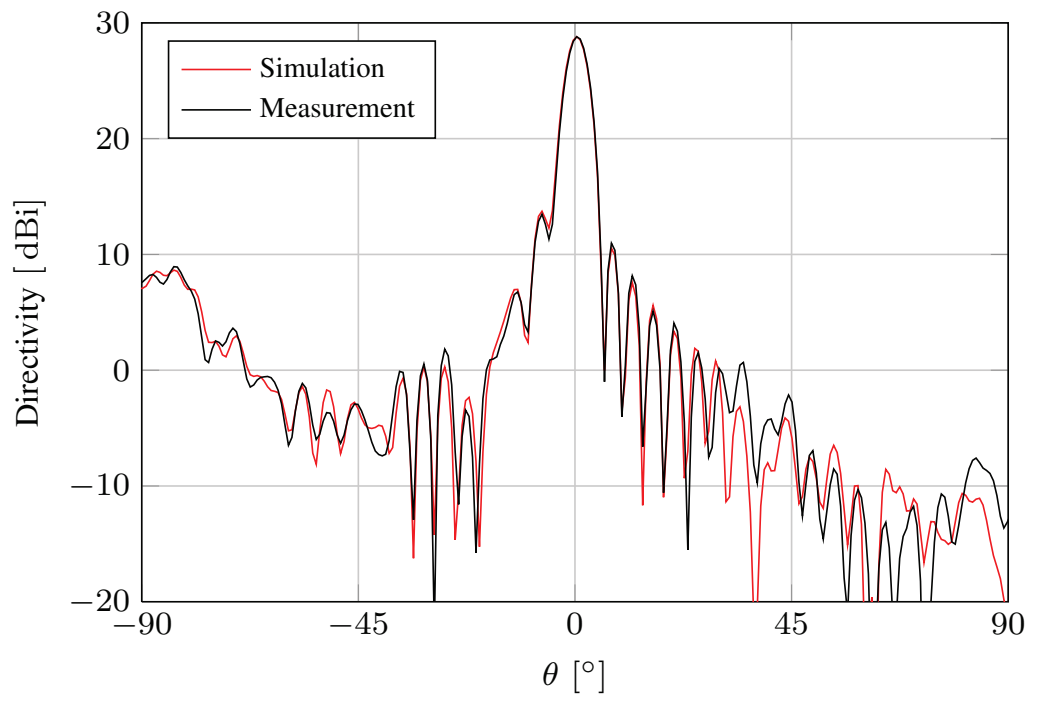

Fig. 2. Comparison of the measured and simulated patterns of the reflectarray sample.

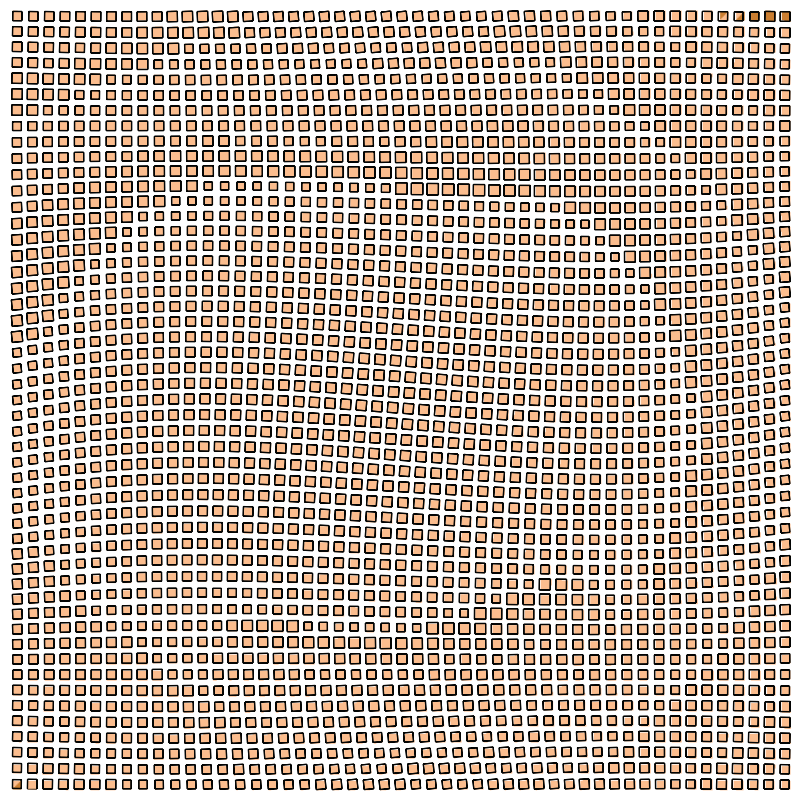

Fig. 3. Reflectarray optimized for a contoured beam coverage, maximizing the gain in Europe and with side-lobe suppression in Southern Africa. The reflectarray elements are arranged in an optimized irregular grid, see [3] for details. 

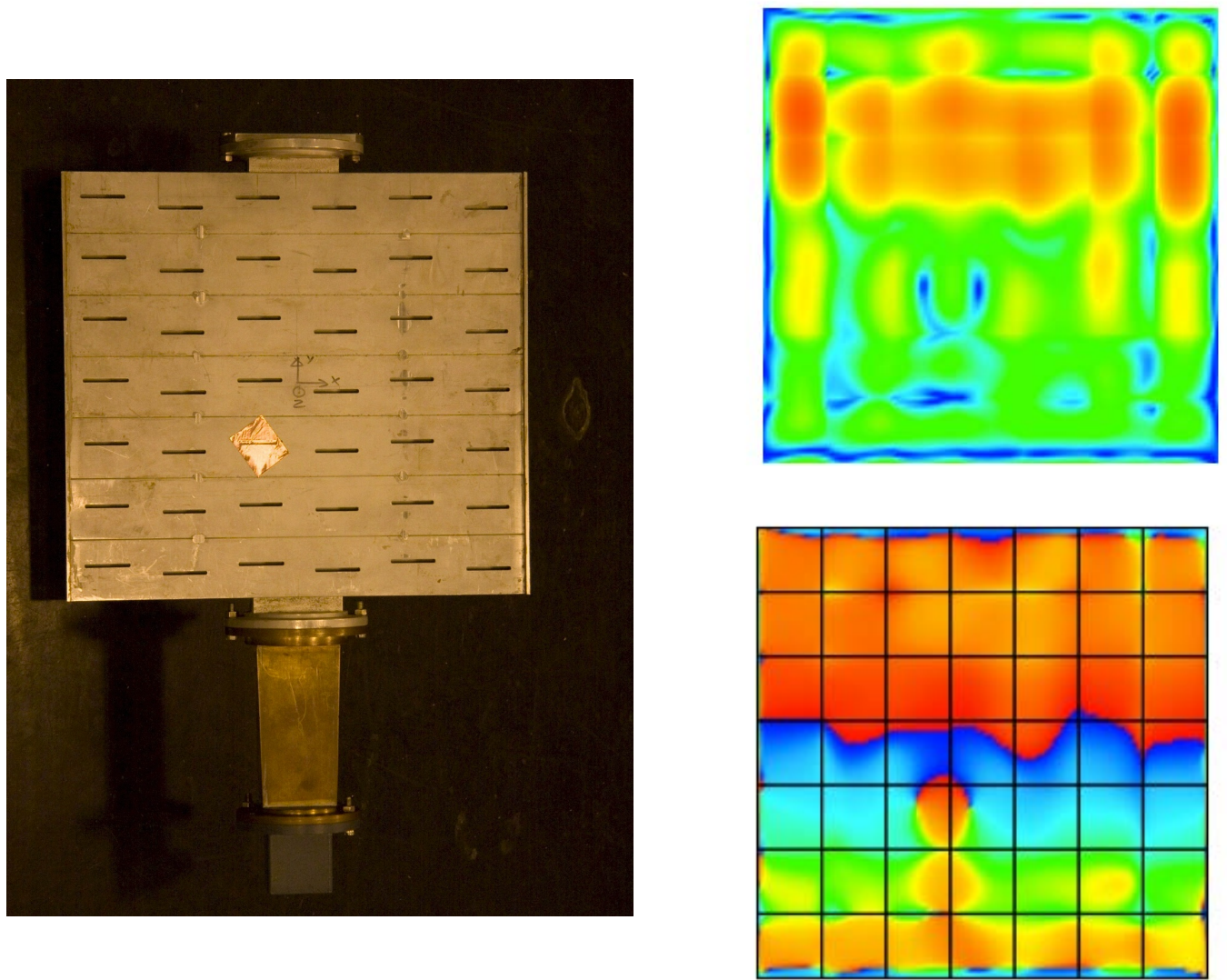

Fig. 4. Slotted waveguide array with an intentionally covered slot and a row-wise problem with the excitation. Left: Photo showing the location of the covered slot. Upper right: Amplitude of the reconstructed co-polar aperture field revealing the location of the covered slot and a too strong excitation of rows 2 and 3 from the top. Lower right: Phase of reconstructed co-polar aperture field showing a distinct phase difference at the location of the covered slot and the slot below. The array and the measurements are provided by the DTU-ESA Spherical Near-Field Test Facility at the Technical University of Denmark.

CHAMP also offers a very powerful optimization framework where the feed geometry can be optimized in an hierarchical way, starting from a high-level model using very few parameters, and ranging to a fine-grained model with a large number of degrees of freedom. CHAMP includes a body-ofrevolution MoM (BOR-MoM) for analysis of the horn exterior and/or a sub reflector. This BOR-MoM code has now been completely re-engineered to greatly enhance the modelling speed and accuracy for compact reflector systems, including a possible dielectric support structure. The new BOR-MoM code uses higher-order hierarchical basis functions [1] and smooth curvilinear patches to accurately represent curved surfaces. The rigorous coupling of the new higher-order BORMoM and CHAMPs existing mode-matching engine leads to a typical runtime of 2-3 seconds for a full-wave model of a compact reflector system. Reflector-shaping based on splines has also been implemented and CHAMP therefore integrates all the required functionality in a single tool. Compact rota- tionally symmetric reflector systems can now be synthesized by simultaneous optimization of the horn profile, dielectric support structure, subreflector shape, and main reflector shape. The desired performance can easily be defined by taking advantage of several built-in optimization goals, e.g., return loss, maximum directivity, side-lobe suppression, or crosspolar radiation.

An example of a compact reflector antenna optimized with CHAMP is shown in Figure 5. The antenna includes a highly shaped main reflector with a diameter of 25 wavelengths, a stepped sub-reflector, and a dielectric support structure. All parameters have been optimized to meet stringent requirements on return loss, side lobes, and cross-polar radiation. 


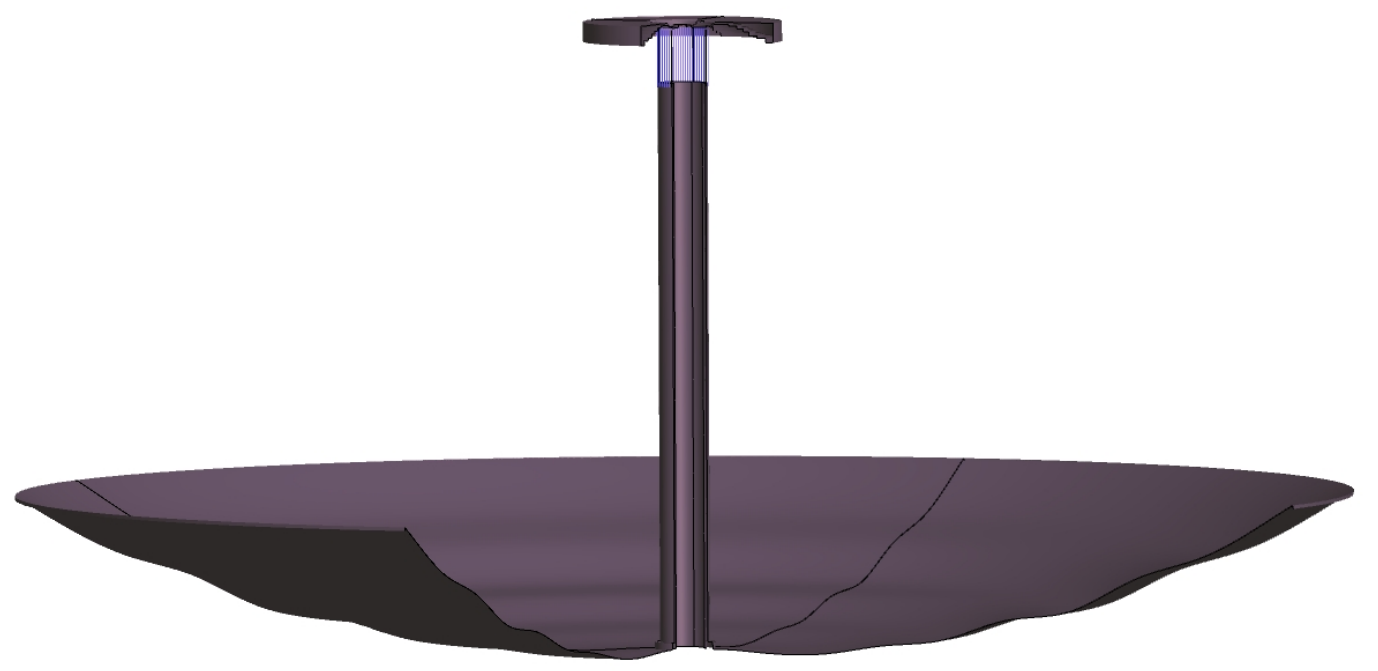

Fig. 5. Optimized rotationally symmetric compact reflector system including a stepped sub-reflector with dielectric support and a highly shaped main reflector.

\section{CONCLUSIONS}

We have described several recent and on-going developments in TICRA's commercial software packages:

- Analysis and design of printed reflectarrays is a major new capability, which is not well supported in currently available commercial software packages. TICRA is now developing a reflectarray design tool combining a newly developed accurate and fast analysis technique with powerful optimization features.

- DIATOOL is a new type of EM modelling software that allows reconstruction of 3D currents and fields on the surface of an antenna, using a measured antenna pattern as input. Typical application areas are antenna diagnostics and pattern filtering by artificial removal of undesired contributions.

- Design of compact rotationally symmetric reflector systems require a fast and accurate full-wave model. TICRA has therefore developed a new higher-order BOR-MoM solver that allows typical systems to be analyzed in a few seconds. The high analysis speed allows numerical optimization of all geometrical parameters, including splinebased shaping of reflector profiles. The updated CHAMP software rigorously combines the new BOR-MoM solver with the existing mode-matching analysis core, which makes CHAMP an ideal design tool for compact reflector antennas such as axially displaced reflectors and reflectors with hat-feeds.

\section{ACKNOWLEDGEMENTS}

Dr. S. Pivnenko and The DTU-ESA Spherical Near-Field Test Facility are acknowledged for providing the measurements of the slot antenna and the reflectarray sample.

\section{REFERENCES}

[1] E. Jørgensen, J. L. Volakis, P. Meincke, and O. Breinbjerg, "Higher order hierarchical Legendre basis functions for electromagnetic modeling," IEEE Transactions on Antennas and Propagation, vol. 52, no. 11, pp. 2985-2995, Nov. 2004.

[2] M. Zhou, S. B. Sørensen, E. Jørgensen, P. Meincke, O. S. Kim, and O. Breinbjerg, "An accurate technique for calculation of radiation from printed reflectarrays," IEEE Antennas Wireless Propag. Lett., vol. 10, pp. 1081-1084, 2011.

[3] M. Zhou, S. B. Sørensen, P. Meincke, E. Jørgensen, O. S. Kim, O. Breinbjerg, and G. Toso, "Design and analysis of printed reflectarrays with irregularly positioned array elements," in Proc. EuCAP, Prague, Czech Republic, 2012

[4] E. Jørgensen, P. Meincke, C. Cappellin, and M. Sabbadini, "Improved source reconstruction technique for antenna diagnostics,", in Proceeedings of the 32nd ESA Antenna Workshop, ESTEC, Noordwijk, The Netherlands, 2010.

[5] E. Jørgensen, P. Meincke, O. Borries, and M. Sabbadini, "Processing of measured fields using advanced inverse method of moments algorithm,,' in Proceeedings of the 33rd ESA Antenna Workshop, ESTEC, Noordwijk, The Netherlands, 2011.

[6] E. Jørgensen, P. Meincke, and C. Cappellin, "Advanced processing of measured fields using field reconstruction techniques," Antennas and Propagation (EUCAP), Proceedings of the 5th European Conference on, pp. $3880-3884,2011$.

[7] E. Jørgensen, D. W. Hess, P. Meincke, O. Borries, C. Cappellin, and J. A. Fordham, "Antenna diagnostics on planar arrays using a 3D source reconstruction technique and spherical Near-Field measurements," in 6th European Conference on Antennas and Propagation (EuCAP 2012), Prague, Czech Republic, Mar. 2012. 